\title{
Why Liver Cancer is so Highly Refractory to Chemotherapy?
}

\section{Marin JJG}

Experimental Hepatology and Drug Targeting (HEVEFARM), University of Salamanca, IBSAL, Salamanca, Spain

*Corresponding author: Jose JG Marin, Department of Physiology and Pharmacology, Campus Miguel de Unamuno E.I.D. S-09, 37007-Salamanca, Spain, Tel: 34-923-294674; Fax: 34-923-294669; E-mail: jjgmarin@usal.es

Rec date: Aug 14, 2015; Acc date: Aug 17, 2015; Pub date: Aug 20, 2015

Copyright: @ 2015 Marin JJG, et al. This is an open-access article distributed under the terms of the Creative Commons Attribution License, which permits unrestricted use, distribution, and reproduction in any medium, provided the original author and source are credited.

\begin{abstract}
In contrast to important advances in early diagnosis of primary liver cancer, both hepatocellular carcinoma (HCC) and cholangiocarcinoma (CCA), and the curative alternatives available to treat these patients, the pharmacological strategies used in adjuvant chemotherapy and in advanced tumors are poorly effective, whereas similar regimes result in much better outcome in other types of cancer. The reason for the marked refractoriness of liver cancer to antitumor drugs, even the newest inhibitors of receptors with tyrosine kinase activity (TKI), is the participation in the overall multidrug resistance (MDR) phenotype of very different mechanisms that are yet poorly understood. This justifies the effort that is being carried out to obtain a complete picture of the question, which will allow us to identify the precise genetic fingerprint accounting for the MDR phenotype present in each tumor at each moment, from diagnosis to the end of treatment. This information shall be valuable to prevent unnecessary use of pharmacological regimes without expected beneficial effect but with potential noxious consequences. Finally, a better understanding of the molecular bases of the problem is also required to develop novel strategies aimed to fight HCC and CCA chemoresistance.
\end{abstract}

Keywords: Cancer; Chemotherapy; Chemoresistance; Cholangiocarcinoma; Hepatocellular carcinomaIntroductionWhy liver cancer is so highly refractory to chemotherapy? The response to this question is complex. In the first place it should be considered that liver cancer is diverse in origin as well as in biological and clinical characteristics. The most frequent type of primary liver cancer is hepatocellular carcinoma (HCC) - derived from hepatocytes - followed at a considerable distance by cholangiocarcinoma (CCA) -derived from epithelial cells of the biliary tree (cholangiocytes) -. Both tumors share a frequent fatal prognosis together with a very poor response to chemotherapy $[1,2]$. The mechanisms accounting for the marked multidrug resistance (MDR) phenotype of HCC and CCA are, in part, similar, but there are also important differences between them [3].During the last few years, several advances in the development of novel drugs against cancer have been achieved. These include the synthesis of a whole family of small molecules able to inhibit tyrosine kinase activity of a variety of receptors involved in several aspects of cancer biology, such as proliferation, angiogenesis, invasion and metastasis [4]. Tyrosine kinase inhibitors (TKIs) have been shown to be effective in the treatment of several types of tumors, but scarcely in patients suffering from primary liver cancer $[5,6]$. Nevertheless, the only drug with recognized beneficial effect in these cases is one of these TKIs, namely sorafenib, and this is only true for a very selected population of HCC patients [7]. However, the beneficial effect of treatment with chemotherapeutic regimes incorporating sorafenib is restricted to prolong their survival only few months [7]. In addition, the use of TKIs, even these (more than 20) approved so far by the US Food and Drug Administration (FDA) and the European Medicines
Agency (EMA), are not free of toxic side effects, including hepatotoxicity, which determines close monitoring during tretament [8]. Other TKIs have been evaluated to treat liver cancer with disappointing results, particularly in phase III trials [7]. The rest of antitumor drugs with different mechanisms of action that have been previously assayed to treat advanced HCC and CCA have resulted in even poorer outcome $[1,2]$. An important goal of modern pharmacology is to characterize the molecular bases of the marked refractoriness of liver cancer to chemotherapy in order to: i) predict the failure of treatment with a given drug or regime and ii) develop novel strategies to overcome this important limitation in the management of these patients. At this respect, several recent studies have shed some light on the question by identifying changes in the expression or the presence of genetic variants in the proteins accounting for liver cancer chemoresistance [9]. Thus, specific transporters accounting for drug uptake have been involved in liver cancer chemoresistance to TKIs. It should be considered that in aqueous solution most TKIs are positively charged ions. This explains why organic actions transporters (OCT) play a key role in TKI uptake, which is required to reach the catalytic region of their target receptors that is located intracellularly. Accordingly, tumor cells that reduce the amount of OCT proteins at their plasma membrane or that express either less functional genetic variants - due to mutations - or inactive truncated forms - due to aberrant splicing - have higher chance of survival under the pharmacological pressure of TKIs. These changes involving OCT1 and OCT3 have been described in HCC and CCA, which may determine a poor response of these tumors to sorafenib [10]. On the other hand, organic anions transporting polypeptides (OATP) are involved in the uptake of anionic drugs. Thus, impaired expression and/or function of OATP1B1 and OATP1B3 in HCC and OATP2A1 in CCA $[3,11]$ may contribute to reduce anticancer drug uptake and hence result in poorer response to chemotherapy in these tumors. High expression of pumps belonging to the ATP-binding cassette (ABC) superfamily of proteins, such as P-glycoprotein or multidrug-resistance protein 1 (MDR1) and several members of the MRP family, are able to actively export a large variety of drugs, such as doxorubicin, etoposide, paclitaxel and vinblastine $[12,13]$, and hence play an important role in decreasing their intracellular levels. Since not only drug concentrations are important, but also the proportion of active molecules reaching tumor cells, mechanisms of chemoresistance found in HCC and CCA include the decreased activation of prodrugs. For instance, impaired activity and/or expression of enzymes involved in the activation of 5'-fluorouracil (5'-FU) and gemcitabine may be involved in the poor response of advanced CCA to the treatment with these drugs $[14,15]$. In the opposite direction, up-regulation of enzymes accounting for drug inactivation, such as several isoforms of glutathione-S-transferase (e.g., GSTP1) [16, 17], whose expression may be stimulate by the pharmacological treatment, also results in reduced response to chemotherapy. An important characteristic that may 
determine the efficacy of chemotherapy is the presence in HCC and CCA cells of changes in the molecular targets for antitumor drugs. This is particularly relevant in the case of TKIs, the appearance of mutations that either induce constitutive activation of the receptors or hinder the potential interaction of these proteins with TKIs, results in the lose of activity of these drugs [6,9]. Enhanced ability of tumor cells to repair damaged macromolecules also results in chemoresistance. For example, the major route to repair 5'-FU-induced misincorporation of fluoronucleotides, i.e., base-excision repair system, is activated in 5'-FU-resistant CCA cells [18]. In the case of agents, such as cisplatin, whose mechanism of action is based on the inactivation of tumor cell machinery for genome replication by the formation of DNA adducts, the enhanced ability of these cells to repair DNA damage permits them to overcome the pharmacological challenge and hence escape apoptosis [19]. In fact, the goal of most antitumor drugs is to stimulate cell death. Accordingly, enhanced expression/function of pro-survival proteins, such as BIRC5, or decreased activity of pro-apoptotic proteins, such as p53, may lead to changes in the balance that determines the escaping of tumor cells from drug-induced apoptosis.In sum, at present, there is a considerable interest in elucidating all mechanisms accounting for HCC and CCA chemoresistance. To obtain a complete picture of the question will allow us to identify the precise genetic fingerprint accounting for the MDR phenotype present in each tumor at each moment from diagnosis to the end of treatment. This information shall be valuable to prevent unnecessary use of regimes without expected beneficial effect but with potential noxious consequences. Finally, a better understanding of the molecular bases of the problem is also required to develop novel strategies aimed to fight HCC and CCA chemoresistance [20].

\section{Financial Support}

This study was supported by the Spanish "Ministerio de Ciencia e Innovación" (SAF2013-40620-R), "Junta de Castilla y León" (SA015U13, BIO/SA65/13 and BIO/SA23/14). The group belongs to the Spanish Network for Cooperative Research on Membrane Transport Proteins (REIT) and CIBERehd.

\section{References}

1. Marin JJ, Romero MR, Briz O (2010) Molecular bases of liver cancer refractoriness to pharmacological treatment. See comment in PubMed Commons below Curr Med Chem 17: 709-740.

2. Marin JJ, Lozano E, Briz O, Al-Abdulla R, Serrano MA, et al. (2015) Molecular Bases Of Chemoresistance In Cholangiocarcinoma. See comment in PubMed Commons below Curr Drug Targets .

3. Martinez-Becerra P, Vaquero J, Romero MR, Lozano E, Anadon C, et al. (2012) No correlation between the expression of FXR and genes involved in multidrug resistance phenotype of primary liver tumors. Mol Pharm 9: 1693-1704.

4. Wu P, Nielsen TE, Clausen MH (2015) FDA-approved small-molecule kinase inhibitors. See comment in PubMed Commons below Trends Pharmacol Sci 36: 422-439.

5. Muntané J, De la Rosa AJ, Docobo F, García-Carbonero R, Padillo FJ (2013) Targeting tyrosine kinase receptors in hepatocellular carcinoma. See comment in PubMed Commons below Curr Cancer Drug Targets 13: 300-312.
6. Rizvi S, Borad MJ, Patel T, Gores GJ (2014) Cholangiocarcinoma: molecular pathways and therapeutic opportunities. See comment in PubMed Commons below Semin Liver Dis 34: 456-464.

7. Kalyan A, Nimeiri H, Kulik L (2015) Systemic therapy of hepatocellular carcinoma: current and promising. See comment in PubMed Commons below Clin Liver Dis 19: 421-432.

8. Shah RR, Morganroth J, Shah DR (2013) Hepatotoxicity of tyrosine kinase inhibitors: clinical and regulatory perspectives. See comment in PubMed Commons below Drug Saf 36: 491-503.

9. Marin JJ, Briz O, Monte MJ, Blazquez AG, Macias RI (2012) Genetic variants in genes involved in mechanisms of chemoresistance to anticancer drugs. Curr Cancer Drug Targets 12: 402-438.

10. Herraez E, Lozano E, Macias RI, Vaquero J, Bujanda L, et al. (2013) Expression of SLC22A1 variants may affect the response of hepatocellular carcinoma and cholangiocarcinoma to sorafenib. See comment in PubMed Commons below Hepatology 58: 1065-1073.

11. Wlcek K, Svoboda M, Riha J, Zakaria S, Olszewski U, et al. (2011) The analysis of organic anion transporting polypeptide (OATP) mRNA and protein patterns in primary and metastatic liver cancer. See comment in PubMed Commons below Cancer Biol Ther 11: 801-811.

12. Hunter J, Hirst BH, Simmons NL (1991) Epithelial secretion of vinblastine by human intestinal adenocarcinoma cell (HCT-8 and T84) layers expressing P-glycoprotein. See comment in PubMed Commons below $\mathrm{Br} \mathrm{J}$ Cancer 64: 437-444.

13. Ambudkar SV, Dey S, Hrycyna CA, Ramachandra M, Pastan I, et al. (1999) Biochemical, cellular, and pharmacological aspects of the multidrug transporter. See comment in PubMed Commons below Annu Rev Pharmacol Toxicol 39: 361-398.

14. Evrard A, Cuq P, Ciccolini J, Vian L, Cano JP (1999) Increased cytotoxicity and bystander effect of 5-fluorouracil and 5-deoxy-5-fluorouridine in human colorectal cancer cells transfected with thymidine phosphorylase. Br J Cancer 80: 1726-1733.

15. Hahnvajanawong C, Chaiyagool J, Seubwai W, Bhudhisawasdi V, Namwat N, et al. (2012) Orotate phosphoribosyl transferase mRNA expression and the response of cholangiocarcinoma to 5-fluorouracil. See comment in PubMed Commons below World J Gastroenterol 18: 3955-3961.

16. Nakajima T, Takayama T, Miyanishi K, Nobuoka A, Hayashi T, et al. (2003) Reversal of multiple drug resistance in cholangiocarcinoma by the glutathione S-transferase-pi-specific inhibitor O1-hexadecyl-gammaglutamyl-S-benzylcysteinyl-D-phenylglycine ethylester. J Pharmacol Exp Ther 306: 861-869.

17. Morsi MI, Hussein AE, Mostafa M, El-Abd E, El-Moneim NA (2006) Evaluation of tumour necrosis factor-alpha, soluble P-selectin, gammaglutamyl transferase, glutathione S-transferase-pi and alpha-fetoprotein in patients with hepatocellular carcinoma before and during chemotherapy. Br J Biomed Sci 63: 74-78.

18. Namwat N, Amimanan P, Loilome W, Jearanaikoon P, Sripa B, et al. (2008) Characterization of 5-fluorouracil-resistant cholangiocarcinoma cell lines. See comment in PubMed Commons below Chemotherapy 54: 343-351.

19. Hwang IG, Jang JS, Do JH, Kang JH, Lee GW, et al. (2011) Different relation between ERCC1 overexpression and treatment outcomes of two platinum agents in advanced biliary tract adenocarcinoma patients. Cancer Chemother Pharmacol 68: 935-944.

20. Marin JJ, Castaño B, Blazquez AG, Rosales R, Efferth T, et al. (2010) Strategies for overcoming chemotherapy resistance in enterohepatic tumours. See comment in PubMed Commons below Curr Mol Med 10: 467-485. 